\title{
Clinical Follow-up on Sagittal Fracture at the Temporal Root of the Zygomatic Arch: Does It Need Open Reduction?
}

\author{
Ji Seon Cheon, Bin Na Seo, Jeong Yeol Yang, Kyung Min Son \\ Department of Plastic and Reconstructive Surgery, Chosun University College of Medicine, Gwangju, Korea
}

Background The zygoma is a major portion of the midfacial contour. When deformity occurs in this area, a reduction should be conducted to correct it. If a sagittal fracture at the temporal root of the zygomatic arch occurs, this also requires reduction, but it is difficult to approach due to its anatomical location, and the possibility of fixation is also limited. Thus, the authors attempted the reduction of sagittal fracture by two- or three-point fixation and the Gillies approach without direct manipulation. The preoperative and postoperative results of the patients were evaluated. Follow-up was performed to establish a treatment guideline.

Methods A retrospective study was done with 40 patients who had sagittal fractures at the temporal root of the zygomatic arch from March 2009 to June 2012. Only two- or three-point fixation was performed for the accompanying zygomatic-orbital-maxillary fracture. The Gillies approach was used for complex fractures of the zygomatic arch, while the temporal root of the zygomatic arch was only observed without reduction. Preoperative and postoperative computed tomography and $\mathrm{X}$-ray scans were performed to examine the results.

Results The result of the paired t-test on preoperative and postoperative bone gap differences, the depression level, and the degree of temporal protrusion showed a marked decrease in the mean difference at a 95\% confidence interval. The results were acceptable.

Conclusions In the treatment of sagittal fractures at the temporal root of the zygomatic arch, it is acceptable to use indirect reduction and non-fixation methods. This leads to a satisfactory aesthetic and functional outcome.

Keywords Zygomatic fractures / Facial asymmetry / Follow-up studies

\author{
Correspondence: Jeong Yeol Yang \\ Department of Plastic and \\ Reconstructive Surgery, Chosun \\ University College of Medicine, 365 \\ Pilmun-daero, Dong-gu, \\ Gwangju 501-717, Korea \\ Tel: +82-62-220-3180 \\ Fax: +82-62-225-0996 \\ E-mail: jyyang@chosun.ac.kr
}

This study was supported by research funds from Chosun University, 2009.

This article was presented at the 70th Congress of the Korean Society of Plastic and Reconstructive Surgeons on November 9-11, 2012 in Seoul, Korea

No potential conflict of interest relevant to this article was reported.

\section{INTRODUCTION}

Accompanying a growth in population and the increase in incidents such as violence and traffic accidents, the frequency of facial bone fractures has also increased. The zygomatic arch, important for midfacial area symmetry and has a protruded structure which is particularly vulnerable to fracture. As a prime point of fracture alignment, it plays an important role in reduction $[1,2]$. Serious functional or aesthetic problems may arise depending on the treatment results. Therefore, an accurate diagnosis, appropriate reduction of fracture fragments, and rigid fixation are required $[3,4]$. In this study, cases of traumatic sagittal fractures at the temporal root of the zygomatic arch, which resulted from severe posterior and lateral displacements, were 
reviewed. The fracture at this area is originated from the zygomatic process of the temporal bone. When a sagittal fracture occurs, reduction may likewise be necessary in other areas. This area, however, is difficult to access, and fixation is limited by the important structures around the area. Accordingly, few studies on sagittal fractures at the temporal root of the zygomatic arch have been performed. Therefore, reported herein is a study on sagittal fractures at the temporal root of the zygomatic arch in patients with an accompanying zygomatic-orbital-maxillary fracture. Using classic two- or three-point fixation, the fracture sites were stabilized. For the zygomatic arch, a closed reduction was performed using a classic Gillies approach, while the sagittal fracture site of the temporal root of the zygomatic arch was clinically observed without open reduction or fixation. A follow-up was done to examine the radiologic decrease in bone gap and bony protrusion in the zygomatic arch sagittal fracture site without reduction and fixation, as well as the contour and symmetry of the zygomatic arch on the intact side. Through this comparative study, the authors evaluated the effect of indirect reduction on the sagittal fracture at the temporal root of the zygomatic arch. This allowed us to propose possible treatment guidelines for sagittal fractures at the temporal root of the zygomatic arch.

\section{METHODS}

\section{Subjects}

Among the patients who underwent reductions in the authors' hospital between March 2009 and July 2012 due to midfacial injuries, 40 patients who showed signs of sagittal fracture at the temporal root of the zygomatic arch were selected for this retrospective study. Patients who had a history of fracture or who had facial asymmetry due to causes other than fracture were excluded from this study.

\section{Methods}

In all cases, surgery was performed under general anesthesia between 5 and 14 days after the injuries were received. In order to expose the fragments of the complex zygomatic-orbital-maxillary fracture site, subciliary, subbrow, temporal, and gingivobuccal approaches were used, while a two- or three-point fixation was performed using absorbable plates (Biosorb, Conmed, NY, USA). Closed reduction was performed using the Gillies approach for complex fractures of the zygomatic arch, while the sagittal fracture site at the zygomatic process of the temporal bone was observed only without performing open reduction and fixation. The sagittal fracture cases at the temporal root of the zygomatic arch were followed up without direct reduction. Postoperative liquid diets were provided to the patients for a week. They were instructed not to eat hard foods but only a soft diet for about two weeks after they were discharged from the hospital. All of the patients were asked to wear arch protectors for three weeks after the surgery to prevent them from receiving additional injuries or damages on the operated sites.

All patients were evaluated preoperative and postoperatively with X-ray and computed tomography (CT) imaging. For the comparison of preoperative and postoperative results, we measured the following 3 items, using the diagnostic images.

\section{The method of measurement using diagnostic images Measurement of the bone gap}

The bone gap was examined through preoperative and postoperative $\mathrm{CT}$ images by measuring the length of the widest portion in the sagittal fracture areas (Fig. 1).

\section{Measurement of the degree of the zygoma depression}

The level of zygomatic depression and facial symmetry were compared through the following method. The normal side shown

\section{Fig. 1. Measurement of bone gap}

(A) A schematization of the preoperative computed tomography of a 63-yearold male patient. The sagittal fracture at the root of the zygomatic arch can be seen (red round). (B) Magnified view. The length of the widest portion of the sagittal fracture site was measured (red arrow).
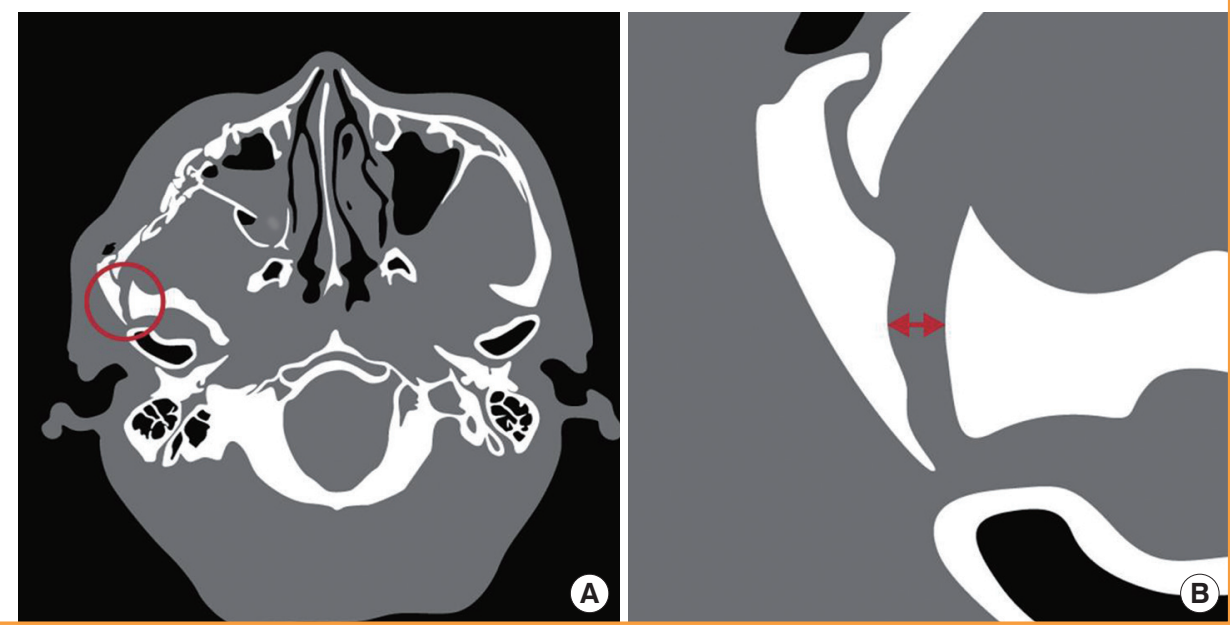


\section{Fig. 2. Measurement of the degree of depression}

After tracing the shadow of the injured zygomatic cortex, the intact side was overturned and overlapped on the affected side in order to measure the distance of the gap (red curve arrow). Then, the vertical distance was measured between the severest depression point and the lateral cortex of the intact bony shadow (red arrow in a circle).

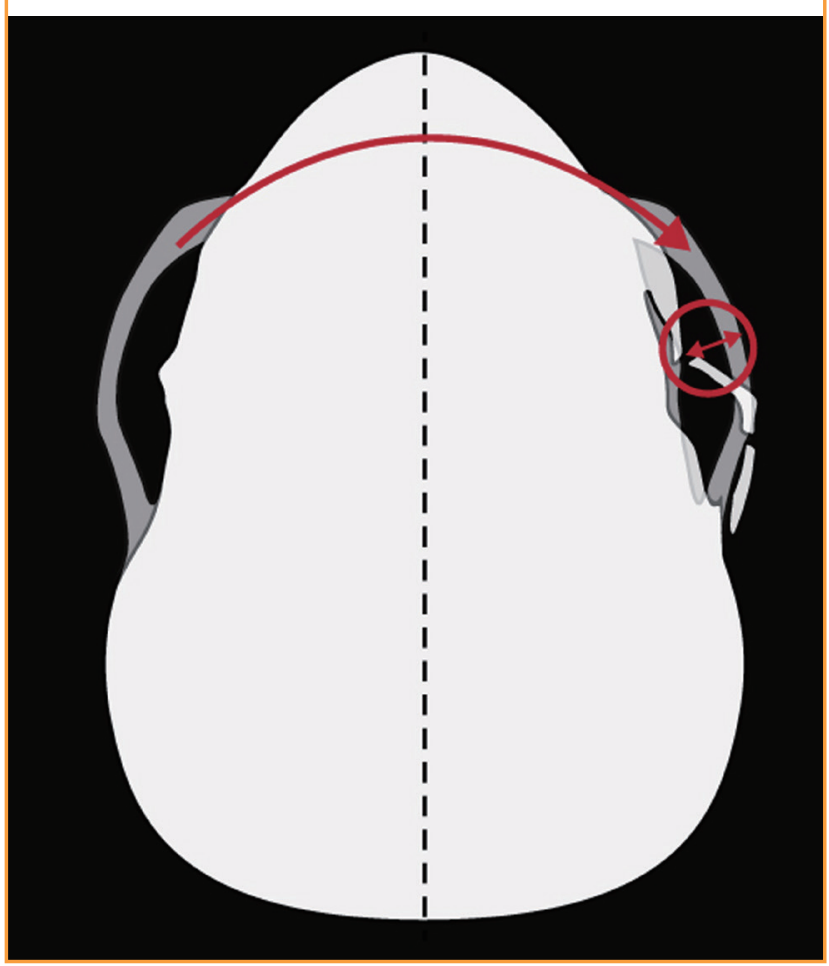

in the preoperative and postoperative $\mathrm{X}$-ray images was transcribed to waxed paper. The paper was overturned, and then was overlapped onto the images of the affected side to compare with the gap by measuring the vertical distance between the severest depression point and the lateral side of the intact bony shadow (Fig. 2) [1].

\section{Measurement of the degree of temporal protrusion}

The degree of temporal protrusion was investigated through the preoperative and postoperative CT images. The vertical distance between the anterior edge of the widest portion on the sagittal fracture site from the sagittal midline was measured. Then, the distance of the non-fractured side was measured. The gap between the above two distances was defined as the temporal protrusion (Fig. 3).

\section{Statistical analysis}

All values were the means of two consecutive measurements. For a mean analysis (t-test), PASW ver. 18 (SPSS Inc., Chicago, IL, USA) was used in a paired t-test. Statistical significance was considered to be when the P-value was less than 0.05 . All of the results are presented as mean \pm standard deviation.

\section{Fig. 3. Measurement of the degree of temporal protrusion}

After marking the anterior edge of the affected side (point a), the vertical distances were measured on both the affected side and non-affected side (point b) from the sagittal midline (point c). The difference in the two distances (ac-bc) was calculated.

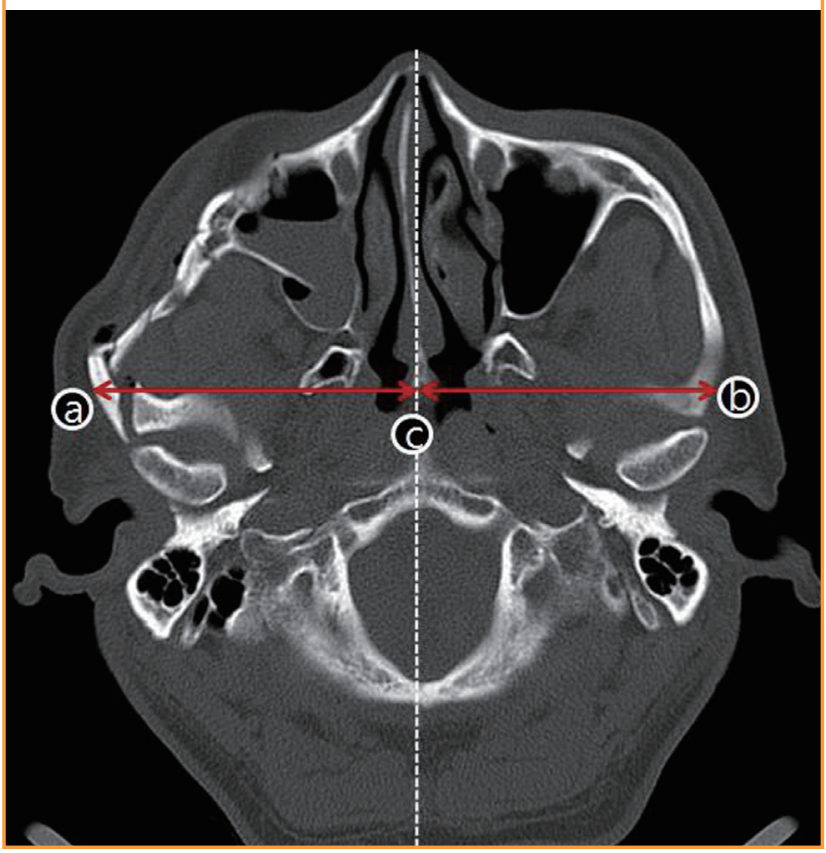

\section{RESULTS}

Among the patients who underwent facial bone fracture surgery, 40 patients were confirmed to have had sagittal fractures at the temporal root of the zygomatic arch. The age of the subjects ranged from 22 to 76 with a mean of 43.4 . There were 34 male subjects and 6 female subjects. There were 22 cases of left sagittal fractures at the temporal root of the zygomatic arch and 18 cases of right sagittal fractures at the temporal root of the zygomatic arch. There were no patients with fractures in both sides. As to the cause of injury, traffic accidents formed the largest portion, with 14 cases; followed by falls, with 12 cases; slipping, with 8 cases; blunt trauma, with 4 cases; and physical assault, with 2 cases. Among the accompanying fractures, tripod fractures formed the largest portion, with 32 cases; followed by orbital floor fractures, with 11 cases; mandible fractures, with 2 cases; and 7 cases of nasoorbitoethmoidal and frontal bone fractures. The shortest follow-up period was one month, and the longest was two years and five months. The average follow-up period was one year and two months (Table 1).

The bone gaps that were measured preoperatively among the sagittal fracture fragments of the zygomatic process of the temporal bone ranged from 1.13 to $5.27 \mathrm{~mm}$. The mean value was $2.24 \pm 1.02 \mathrm{~mm}$. The bone gaps measured at a mean of 1 year and 2 months after the open reduction of the zygomatic-orbital- 


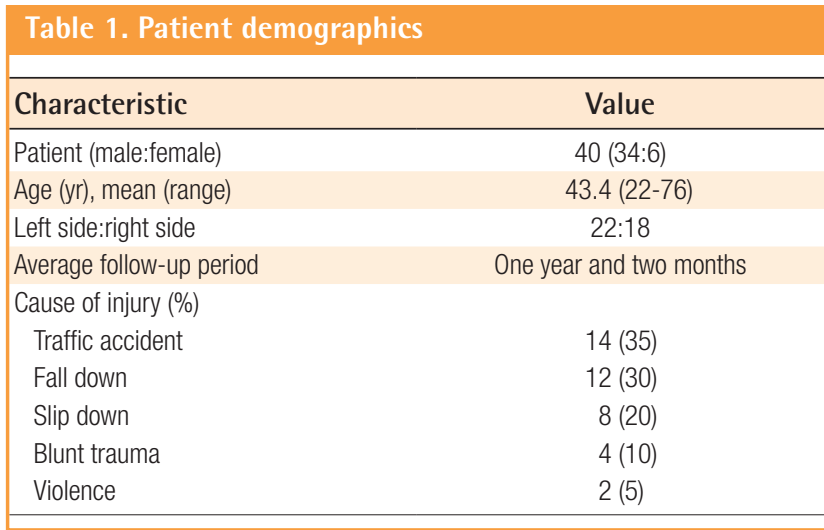

maxillary fracture under general anesthesia, and after the closed reduction using the Gillies approach for the zygomatic arch ranged from 0.2 to $2.56 \mathrm{~mm}$. The mean value was $1.21 \pm 0.55$ $\mathrm{mm}$. The mean difference of the bone gap was $1.03 \pm 0.05 \mathrm{~mm}$ (Table 2).

The level of the zygomatic depression, which was measured to assess facial symmetry by comparing it with the non-affected side, decreased from a preoperative mean of $4.04 \pm 2.70 \mathrm{~mm}$ to a postoperative mean of $2.64 \pm 1.11 \mathrm{~mm}$. The mean difference of the zygoma depression was $1.40 \pm 1.26 \mathrm{~mm}$ after surgery (Table 2).

The degree of temporal protrusion, which was measured to assess facial symmetry by comparing it with the non-affected side, decreased from a preoperative mean of $3.08 \pm 2.08 \mathrm{~mm}$ to a postoperative mean of $0.86 \pm 1.35 \mathrm{~mm}$. It showed a mean difference of $2.22 \pm 1.46 \mathrm{~mm}$ after surgery (Table 2).

The results of the paired $t$-test on the preoperative and postoperative bone gap differences, the depression level and the degree of temporal protrusion showed a marked decrease in the mean difference with a 95\% confidence interval (Table 2).

Among the 40 subjects, 29 showed an accurate reduction of under $2 \mathrm{~mm}$ and 3 showed a minimal depression $(2-3 \mathrm{~mm})$. No apparent deformation was observed, either in the 8 subjects who reported a 3-mm ( 2 cases) or more depression ( 4 cases), or in the subjects who reported overcorrections $(<-0.05 \mathrm{~mm})(2$ cases). In all, there were no specific patient complaints reported and patient compliance was acceptable. No particular complications (e.g., postoperative bleeding, hematoma, infection, facial nerve injury, limitation of mouth motion, malocclusion, facial contour deformation, wound dehiscence, non-union, or temporomandibular joint problems) were observed in the patient group other than facial numbness, which was seen in 8 patients. The patients did not complain of aesthetic or functional problems during the follow-up period, and were mostly satisfied with the outcomes of their operation.
Table 2. Statistical analysis of the preoperative and postoperative bone gap, depression, and protrusion $(n=40)$

\begin{tabular}{|c|c|c|c|}
\hline Variable & Preoperative & $\begin{array}{l}\text { Postopera- } \\
\text { tive }\end{array}$ & $\begin{array}{c}\text { Mean } \\
\text { difference }\end{array}$ \\
\hline Degree of bone gap & $2.24 \pm 1.02$ & $1.21 \pm 0.55$ & $1.03 \pm 0.05$ \\
\hline $95 \% \mathrm{Cl}$ & $1.77-2.43$ & $1.03-1.39$ & $0.67-1.34$ \\
\hline P-value & $0.000^{\mathrm{a})}$ & $0.000^{\mathrm{a})}$ & $0.000^{\mathrm{b})}$ \\
\hline Degree of depression & $4.04 \pm 2.70$ & $2.64 \pm 1.11$ & $1.40 \pm 1.26$ \\
\hline $95 \% \mathrm{Cl}$ & $3.85-7.22$ & $0.42-8.89$ & $1.27-4.36$ \\
\hline P-value & $0.000^{\mathrm{a})}$ & $0.032^{\mathrm{a})}$ & $0.000^{\mathrm{b})}$ \\
\hline Degree of protrusion & $3.08 \pm 2.08$ & $0.86 \pm 1.35$ & $2.22 \pm 1.46$ \\
\hline $95 \% \mathrm{Cl}$ & $2.05-4.11$ & $0.07-1.64$ & $1.40-3.04$ \\
\hline P-value & $0.000^{\mathrm{a})}$ & $0.033^{\mathrm{a})}$ & $0.000^{b)}$ \\
\hline
\end{tabular}

\section{DISCUSSION}

The zygoma is a structure that protrudes from the center and the side of the face forming the malar eminence and connects to the frontal, maxillary, temporal, and sphenoid bones, determining facial symmetry and contour. Due to its protruded shape, the bone is vulnerable to injuries, and zygomatic fracture forms a major portion of facial fractures $[4,5]$. In particular, since the zygomatic arch is a horizontal bone on the side of the face, precise assessment of its position is important in the acute repair of mid-facial fractures [3]. This bone is formed by the temporal process of the zygomatic bone (anterior two-thirds) and the zygomatic process of the temporal bone (posterior one-third). In addition, the posterior end of the zygomatic arch is attached to the squamous part of the temporal bone by anterior and posterior roots $[6,7]$.

There are various types of arch fractures in zygomatic bone fractures. The zygomatic arch fractures can be divided into the medial displacement of the arch, the lateral displacement of the arch (simple fracture), the lateral displacement of the arch (comminuted fracture) and the lateral displacement of the arch (comminuted fracture combined with root fracture) [8]. Among these types of fractures, the authors were interested in the sagittal zygomatic arch fracture, which occurs in the zygomatic process, originating from the squamous part of the temporal bone. When a traumatic fracture develops at the zygomaticotemporal suture line, the fracture is often sagittal to the temporal articulation area, and in this case, it was reported as a sagittal fracture at the root of the zygomatic arch [2]. Gruss et al. [3] divided zygomatic fractures into those of the zygomatic body and the zygomatic arch and then subdivided them. According to their classification, a sagittal fracture can be a combination of lateral displacement and outward bowing or comminuted forms of the zygomatic arch, which is accompanied by various types of zy- 
gomatic body fractures. These sagittal fractures usually develop when severe external force is directly applied to the zygomatic complex and the weaker bones in the zygoma articulation region are fragmented and absorbed, leading to displacement, rotation of bony fragments, and an apparent increase in facial width [7]. Because the zygomatic arch functions as the prime point of alignment in a selective zygomatic-orbital-maxillary fracture with severe posterior or lateral displacement, the fracture at the root of the zygomatic arch must first be reduced for appropriate anterior-posterior and lateral projection of the zygoma [2]. That is, since the type and pattern of zygomatic arch fractures are important in midfacial reconstruction, these arch fractures should be further subdivided within this classification system to reflect the various possible combinations of both coexisting and isolated body and arch fractures in order to plan a therapeutic approach. Therefore, the consideration of new categories for this nomenclature can be viewed as a valuable endeavor.

But, in the cases in this study, which were sagittal fractures occurring in the zygomatic arch area, accessibility to the fracture site as well as fixation were difficult due to nearby structures such as the facial nerve (temporal branch), auriculo-temporal nerve, temporomandibular joint, cranial base, and external auditory canal. No protocol for the fixation and reduction of a zygomatic fracture has been established yet, but clinicians have introduced various approaches and fixation methods that consider postoperative stability and bone union [9]. In particular, for comminuted malar fractures of the zygomatic arch, rigid internal fixation is often required. For complete access to the entire horizontal arc of the zygomatic arch, a bicoronal approach, preauricular approach, modified temporal incision, or an endoscopic temporal approach can be used [2,6,10-12]. Fixation of the arch to the temporal bone also allows for accurate reduction through these approaches [4]. Previous studies have recommended invasive methods for fractures at the temporal root of the zygomatic arch $[2,8]$. Nevertheless, these invasive approaches result in massive bleeding, hair loss at the incision site, scarring, persistent postoperative edema, temporal depression, and numbness in the temporal branch of the facial nerve distribution area and the auriculotemporal nerve distribution area. In addition, postoperative complications such as damage in extensively dissected soft tissue and supporting ligaments, as well as sagging cheeks were possible. Moreover, since sagittal fractures are greenstick fractures, fragments cannot be easily found when approaching from the outside, and fixation is difficult $[10,11]$. When fixation is performed using a microscrew by the previously reported method, the inadvertent displacement of the screw may develop in the superior aspect of the temporomandibular joint or external auditory canal. Screws, when protruding into the sinus cavity, can induce an inflammatory reaction, with granulation tissue formation leading to the loosening of the screws and bony resorption. Also, a simple screw fixation may not be enough to maintain the appropriate supporting strength [2]. Extensive plating of the zygomatic arch area may lead to unwanted results, such as the inaccurate restoration of zygoma configuration, an inflammatory reaction, and bone fragment resorption during the process of miniplate osteosynthesis. Once the other fracture sites are correctly reduced and fixed, the arch is usually splinted by adjacent structures [13]. Therefore, instead of these invasive treatments, we used typical two- or three-point fixation and the Gillies approach without direct reduction and fixation at the sagittal fracture site. As a result, a slight reduction in the bone gap at the sagittal fracture site of the zygomaticotemporal area, relatively acceptable postoperative facial symmetry and a decrease in the temporal protrusion were obtained (Fig. 4). Ha et al. [1] noted that the average depth of zygomatic depression after the Gilles approach was $2.3 \mathrm{~mm}$ (range, 1-6 mm). According to our research, the comminuted zygoma fractures without sagittal fracture showed $2.27 \mathrm{~mm}$ (range, 0.85-5.46 mm) postoperative depression and those with sagittal fracture showed 2.64 $\mathrm{mm}$ (range, $0.05-8.57 \mathrm{~mm}$ ). The increased degree of zygoma depression is thought to be caused by the sagittal fracture.

One of the most important factors in postoperative deformations is the force that pulls the masseter muscle inwardly and downwardly [14]. Taking this force into consideration, masticatory movement was minimized by providing the subjects with a liquid diet for a week after surgery and with sufficient patient education. As a result, successful outcomes were obtained without fragment displacement during the follow-up period. Nevertheless, since the methods used in this study cannot accomplish rigid fixation, recurrent fragment displacement may develop if a traumatic impact is applied, or if the patient does not comply with the directions given preoperatively. The methods used in this study have additional limitations. Even though an indirect reduction and non-fixation are planned prior to surgery, an accurate reduction level cannot be measured during the surgery. This indirect method is not applicable if the stability of the fragments deteriorate after the reduction.

When the method of this study was compared with the typical invasive methods (bicoronal approach, etc.), comparatively acceptable results were obtained with a short operation time. Moreover, various complications caused by extensive incision and dissection or complications caused by the plate and screw, which is a direct fixation method for the sagittal fracture site of the root of the zygomatic arch, can be avoided through the method proposed in this study. The extensive invasive approach was widely used in the past, but these days, aesthetic factors have 


\section{Fig. 4. A 63-year-old man with a sagittal fracture}

(A) Preoperative computed tomography (CT) view. Lateral displacement and outward bowing of the zygomatic arch was found, accompanied by a sagittal fracture at the zygomatic process of the temporal bone. (B) Postoperative 18 month follow-up CT scan demonstrating anatomic restoration of the comminuted arch fracture and a remarkably decreased bone gap and temporal protrusion on the sagittal fracture site. (C) Preoperative worm's eye view shows prominent bone temporal protrusion compared with the intact side. (D) After reduction, comparatively acceptable postoperative facial symmetry and a decrease in temporal protrusion were seen (postoperative 18 month view).
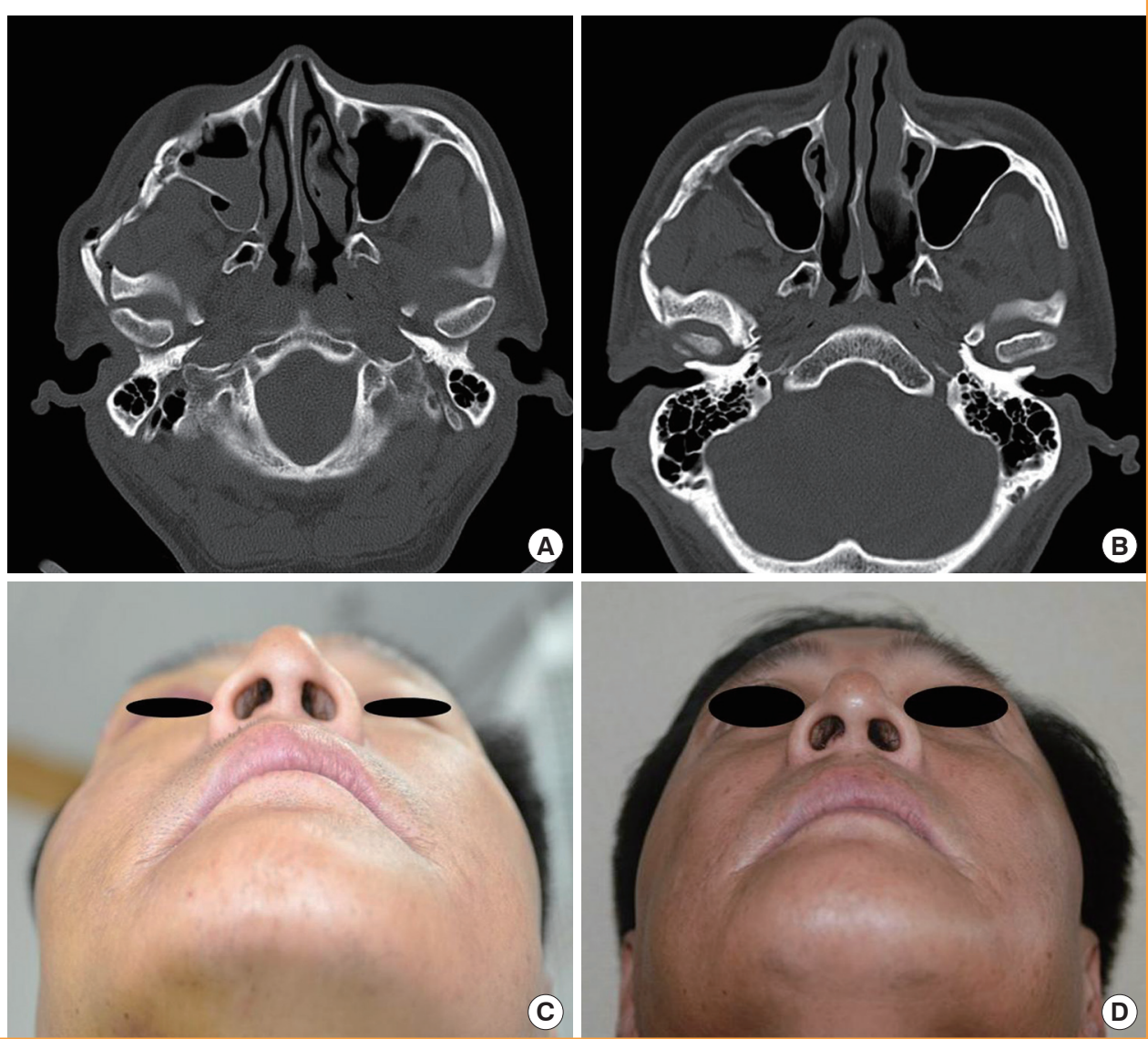

gained in importance. Thus, the selective exposure of the buttress and a non-invasive reduction are also used $[10,15]$. In terms of postoperative patient satisfaction, not only the skeletal structure but also the soft tissue that covers the structure are important [8]. In most zygomatic fracture cases, a rigid fixation using anatomical reduction and multi-fixation has generally been recognized. However, we can obtain acceptable results without complications in cases of sagittal fractures occurring at the temporal root of the zygomatic arch without using direct fixation but rather indirect reduction through reduction and fixation at other accompanying fracture sites. Through the method proposed in this study, comparatively satisfactory fragment reduction (a significant reduction of the bone gap and temporal protrusion) was obtained. With respect to postoperative facial symmetry, relatively satisfactory results were obtained with no patient complaints, even though mild depression and over-correction were observed in some patients. It was found that sideburns concealed mild deformation of the posterior temporal root area. Also, during the follow-up period of one year and two months, patients could perform physiological masticatory movements satisfactorily without fracture dislocation. These conclusions indicate that comparatively satisfactory outcomes can be obtained without direct reduction and fixation of sagittal fractures at the temporal root of the zygomatic arch if appropriate reduction and fixation are performed for the accompanying zygomatic-orbitalmaxillary fracture, and if postoperative management is properly conducted. Finally, it is expected that a more thorough understanding could be gained if prospective studies based on clinical trials in which indirect reduction of this area is compared with a control group undergoing invasive approaches. Many more cases must be carried out in order to establish the efficiency and the efficacy of this indirect method.

\section{REFERENCES}

1. Ha SI, Kim JB, Choi JK, et al. A long term follow up of reduced isolated zygomatic arch fracture by Gillies' approach. J Korean Soc Plast Reconstr Surg 1998;25:76-84.

2. Stevens MR, Menis MA. Microscrew fixation of zygomatic arch fractures. J Oral Maxillofac Surg 1993;51:1158-9.

3. Gruss JS, Van Wyck L, Phillips JH, et al. The importance of the zygomatic arch in complex midfacial fracture repair and correction of posttraumatic orbitozygomatic deformities. Plast Reconstr Surg 1990;85:878-90. 
4. Evans BG, Evans GR. MOC-PSSM CME article: zygomatic fractures. Plast Reconstr Surg 2008;121:1-11.

5. Hollier LH, Thornton J, Pazmino P, et al. The management of orbitozygomatic fractures. Plast Reconstr Surg 2003;111: 2386-92.

6. Prakasam M, Dolas RS, Managutti A, et al. A modified temporal incision: an alternative approach to the zygomatic arch. J Maxillofac Oral Surg 2010;9:428-33.

7. Stanley RB Jr. The zygomatic arch as a guide to reconstruction of comminuted malar fractures. Arch Otolaryngol Head Neck Surg 1989;115:1459-62.

8. Kang NH, Song SH, Choi SM, et al. Clinical Experiences of Facial Asymmetries in Zygomaticomaxillary Complex Bone Fracture Patients. J Korean Soc Plast Reconstr Surg 2011;38:161-5.

9. Davidson J, Nickerson D, Nickerson B. Zygomatic fractures: comparison of methods of internal fixation. Plast Reconstr Surg 1990;86:25-32.
10. Lee CH, Lee C, Trabulsy PP, et al. A cadaveric and clinical evaluation of endoscopically assisted zygomatic fracture repair. Plast Reconstr Surg 1998;101:333-45.

11. Zhang QB, Dong YJ, Li ZB, et al. Coronal incision for treating zygomatic complex fractures. J Craniomaxillofac Surg 2006;34:182-5.

12. Al-Kayat A, Bramley P. A modified pre-auricular approach to the temporomandibular joint and malar arch. Br J Oral Surg 1979; 17:91-103.

13. Zingg M, Laedrach $K$, Chen J, et al. Classification and treatment of zygomatic fractures: a review of 1,025 cases. J Oral Maxillofac Surg 1992;50:778-90.

14. Rinehart GC, Marsh JL, Hemmer KM, et al. Internal fixation of malar fractures: an experimental biophysical study. Plast Reconstr Surg 1989;84:21-5.

15. Kurita M, Okazaki M, Ozaki M, et al. Patient satisfaction after open reduction and internal fixation of zygomatic bone fractures. J Craniofac Surg 2010;21:45-9. 